\title{
A Neutron Diffraction Study of Selenious Acid, $\mathrm{H}_{2} \mathrm{SeO}_{3}$
}

\author{
F. KREBS LARSEN,* M. S. LEHMANN and INGER SøTOFTE**
}

Department of Inorganic Chemistry, University of Aarhus, DK-8000 C, Denmark

\begin{abstract}
A neutron diffraction study of selenious acid, $\mathrm{H}_{2} \mathrm{SeO}_{3}$, has been carried out. The cell is orthorhombic, space group $P 2_{1} 2_{1} 2_{1}$ (No. 19), with $a=9.132 \AA, b=5.988 \AA$, and $c=5.091 \AA$, and with four formula units per unit cell.

A three-dimensional set of data was collected, giving 591 symmetry independent, significant reflections. Positional and anisotropic temperature factors for all atoms were refined by the method of least squares, leading to a final $R$-value of 0.036 .

The scattering length of selenium was determined to be $(0.810 \pm$ $0.005) \times 10^{-12} \mathrm{~cm}$.

The arrangement of the $\mathrm{SeO}_{3}$ groups previously determined by Wells and Baily is confirmed, and the hydrogen-bond system is described in detail.

The hydrogen atoms seem to be positioned in single potential minima in the hydrogen bonds.
\end{abstract}

\footnotetext{
The structure of selenious acid has previously been determined by Wells and Bailey, ${ }^{1}$ who describe the ordering of the selenite groups, $\mathrm{SeO}_{3}$, in twodimensional hydrogen bonded double layers. Since only a few mineral acids have previously been investigated by neutron diffraction, we found it worthwhile to carry out such an analysis to determine the hydrogen positions in the hydrogen bonds.

An $\mathrm{X}$-ray diffraction analysis of $\mathrm{KH}_{3}\left(\mathrm{SeO}_{3}\right)_{2}$ has been carried out in this Laboratory by Hansen et al., ${ }^{2}$ and at the moment a neutron diffraction analysis is in progress. It is the aim of a forthcoming article to compare the hydrogen bonding system in the two structures.
}

\section{EXPERIMENTAL}

Single crystals of selenious acid were obtained by slow evaporation of a solution, made by dissolving selenium in concentrated nitric acid.

\footnotetext{
* Present address: State University of New York, Chemistry Department, Crystallographic Laboratory, Acheson Hall, Buffalo, New York 14214, USA.

** Present address: Chemical Laboratory B, Technical University of Denmark, DK-2800 Lyngby, Denmark.
} 
The crystals big enough for neutron measurements were irregularly shaped, and for that reason the crystal used for data collection was ground to a cylinder on a small lathe, using a diamond tool.

The cylinder axis was parallel to the c-axis of the crystal, and the dimensions were: diameter $3.4 \mathrm{~mm}$; length $6.8 \mathrm{~mm}(\sigma=0.1 \mathrm{~mm})$.

For data collection, the crystal was mounted in a thinwalled aluminium container.

The cell parameters were redetermined by X-ray powder film-data. A de WolffGuinier camera was employed, using $\mathrm{Cu} K \alpha$ radiation $(\lambda=1.5405)$ and germanium powder $(a=5.6576)$ as reference. 14 lines in the powder diagram were indexed, using the cell parameters given by Wells and Bailey. A new set of cell dimensions was calculated by a least squares program $\mathrm{S}_{1}^{3}$ with the assumption of an orthorhombic crystal system; and by an iterative procedure of indexing and least squares determinations a final number of 35 lines was indexed, giving the following cell parameters:

$$
a=9.132(3), b=5.988(1), c=5.091 \text { (1) } \AA
$$

Numbers in parenthesis are standard deviations in unit of the last digit, as given by the least squares determination.

A three-dimensional set of neutron data was collected on an automatic Hilger-Ferranti four-circle diffractometer, located at the DR 3 reactor at the Danish Atomic Energy Commission Research Establishment, Risø.

The wavelength of the monochromatic neutron beam was $1.025 \AA$, and the flux at the specimen was $0.9 \times 10^{\circ} \mathrm{n} / \mathrm{cm}^{2} / \mathrm{sec}$. The beam ranging over the crystal was uniform within $\pm 5 \%$.

The crystal was mounted with the c-axis parallel to the $\varphi$-axis of the instrument. In an attempt to average out the effect of the double-Bragg scattering, the crystal was rotated an angle $\psi$ about the diffraction vector from the $A$-setting position (Arndt and Willis $\left.{ }^{4}\right), \psi$ being different for symmetry related reflections. The possible values for $\psi$ were $\pm 3^{\circ}, \pm 2^{\circ}$, and $\pm 1^{\circ}$.

The reflections were measured by the $\omega-2 \theta$ scan technique. The Hilger-Ferranti diffractometer scans the reflection in steps, and counts for each step were recorded. The total range of measurement for a reflection was $4.8^{\circ}$. Within $\pm 1.6^{\circ}$ from the expected center of the peak, the size of the step was $0.04^{\circ}$, whereas the size of the step outside this limit was $0.16^{\circ}$. The reflections were recorded in sequence of increasing $\sin \theta / \lambda$. Two standard reflections $5,4,3$ and $3,2,1$ were measured at intervals of 10 and 15 reflections, respectively.

All reflections with $l>0$ and $\sin \theta / \lambda \leq 0.762$ were measured, giving a total of 2220 .

The profile measurements of the reflections were reduced to structure factors by a program DRAM, ${ }^{5}$ which determines the position of the peak and calculates the Lorentz corrected intensities. The estimated standard deviation $\sigma F^{2}$ count is based on counting statistics.

The structure factors were corrected for drift in the experimental conditions, which is reflected in the variation of the intensities of the standard reflections. This variation is of the order of $2 \%$, within a reactor operation period of three weeks.

After averaging over symmetry related reflections, the number of symmetry independent reflections was 612 . Of these, 4 were later found to contain errors originating from malfunction of the instrument, leaving a set of 608 reflections. Of these, only 17 had $F^{2}$ less than $2 \times \sigma F^{2}$ count.

The correction for absorption was calculated, using a programme written by Wells, ${ }^{6}$ assuming that the reflections were recorded in the $A$-setting. The crystal cylinder was described by 18 planes, 16 of these forming a column around the cylinder axis with the remaining 2 planes intersecting at right angles, the lines of intersection forming two regular 16-gons.

The linear absorption coefficient was determined by measurement to be $\mu=1.20 \mathrm{~cm}^{-1}$ $\left(\sigma=0.01 \mathrm{~cm}^{-1}\right)$. 


\section{STRUCTURE REFINEMENT}

A first refinement, using the method of Bhuiya and Stanley, ${ }^{7}$ was performed with the programme D445. ${ }^{8}$ The positions for oxygen and selenium atoms given by Wells and Bailey were refined, and a Fourier synthesis was calculated, phased on the obtained positions. From this Fourier synthesis, the two hydrogen positions were determined.

Further refinements were carried out using the two least squares programmes ORFLS ${ }^{9}$ as found in the X-ray $63^{10}$ system, and G403.11 with

G403 is a block-diagonal program, where the weighting of the form $1 /(\mu F)^{2}$

$$
\mu F=\sqrt{\sigma F_{\text {count }}^{2}+k\left|F_{\mathrm{o}}\right|^{2}}-\left|F_{\mathrm{o}}\right|
$$

can be adjusted after each cycle of refinement by changing $k$. A correction for isotropic extinction following the formula of Zachariasen, ${ }^{12}$ as modified by Larson: ${ }^{13}$

$$
F_{\mathrm{c}}^{*}=K \times F_{\mathrm{c}}\left(1+g \beta(2 \theta) F_{\mathrm{c}}{ }^{2}\right)^{-\frac{1}{2}}
$$

can be included in the refinement. $K$ is the scale factor, and $\beta(2 \theta)$ is given by $A\left(\mathrm{~d} A^{*} / \mathrm{d} \mu\right) / \sin (2 \theta)$, where $A\left(\mathrm{~d} A^{*} / \mathrm{d} \mu\right)$ is obtained from the absorption correction calculations. ( $A^{*}=A^{-1}$ is the absorption factor.)

A first refinement indicated the presence of extinction. To obtain a good estimate for the scale factor and the $g$ factor, a refinement using the 290 weakest reflections was therefore carried out, leading to an $R$ value of 0.073 .

Further refinements of positional and anisotropic temperature parameters plus the extinction factor were performed, leading to an $R$ value of 0.0362 . Structure factors with $F_{0}^{2}$ less than $2 \times \sigma F^{2}$ count were excluded from the refinement.

The scattering lengths used were $b_{\mathrm{H}}=-0.372 \times 10^{-12} \mathrm{~cm}$ (the Neutron Diffraction Commission ${ }^{14}$ ), $b_{\mathrm{O}}=0.588 \times 10^{-12} \mathrm{~cm}$ (Brown and Chidambaram ${ }^{15}$ ), and $b_{\mathrm{Se}}=0.78 \times 10^{-12} \mathrm{~cm}$ (Columinas ${ }^{16}$ ). Other values for $b_{\mathrm{Se}}$ are given by Andresen ${ }^{17}\left(0.86 \times 10^{-12} \mathrm{~cm}\right)$, and by Fuess and Will ${ }^{18}\left(0.85 \times 10^{-12} \mathrm{~cm}\right)$. As these values for $b_{\mathrm{se}}$ differ somewhat, an attempt to determine it from this experiment was done.

The extinction correction obtained from the last refinement was applied to the structure factors, and a refinement on all parameters plus the site occupation factor of the selenium atom was carried out by ORFLS, leading to a scattering length of $0.809 \times 10^{-12} \mathrm{~cm}$. Redetermination of the extinction factor $g$ by refinement with G403, using the obtained $b_{\text {se }}$, and a further refinement with ORFLS led to a final value for $b_{\mathrm{Se}}$ of $0.810 \times 10^{-12} \mathrm{~cm}(\sigma=$ $0.005 \times 10^{-12} \mathrm{~cm}$ ). The $R$ factor was 0.0356 , and the $g$ factor $1.62 \times 10^{-3}$.

The value of $k$ in the weighting expression was set to values in the range 0.030 to 0.045 . In the last cycle of refinement it was 0.045 .

The changes in positional and temperature parameters caused by the change in the scattering length were at maximum one standard deviation, except for the selenium atom. For this atom, the temperature coefficients $u_{11}, u_{22}$, and $u_{33}$ increased (by $0.0014,0.0014,0.0015 \AA^{2}$, respectively). The correlation coefficients between $b_{\mathrm{Se}}$ and $u_{\mathrm{ii}}$ for selenium were of the magnitude 0.38 .

Acta Chem. Scand. 25 (1971) No. 4 


\section{CRYSTAL DATA}

Crystal system: orthorhombic.

Unit cell: $a=9.132$ (3) $\AA, b=5.988$ (1) $\AA, c=5.091$ (1) $\AA$.

Space group: $1 P 2_{1} 2_{1} 2_{1}$ (No. 19).

$D_{x}=3.07 \mathrm{~g} / \mathrm{cm}^{3}$.

There are four formula units in the unit cell. The parameters obtained are given in Table 1. Distances and angles within the selenious acid molecule are given in Table 2 , and in Table 3 distances and angles in the hydrogen bond are summarized.

Table 1a. Positional coordinates.

\begin{tabular}{lccc} 
& \multicolumn{1}{c}{$x$} & $y$ \\
$\mathrm{Se}$ & $0.7943(1)$ & $0.7434(1)$ & $0.3890(2)$ \\
$\mathrm{O}(1)$ & $0.8765(1)$ & $0.8695(2)$ & $0.1431(3)$ \\
$\mathrm{O}(2)$ & $0.9200(2)$ & $0.8200(3)$ & $0.6283(3)$ \\
$\mathrm{O}(3)$ & $0.8431(1)$ & $0.4635(2)$ & $0.3552(3)$ \\
H(1) & $0.8830(3)$ & $0.8021(5)$ & $0.8091(5)$ \\
H(2) & $\mathbf{0 . 9 5 2 2 ( 2 )}$ & $\mathbf{0 . 4 3 9 8}(4)$ & $0.3423(6)$
\end{tabular}

Table 1b. Anisotropic temperature factors in $\AA$.

\begin{tabular}{|c|c|c|c|c|c|c|}
\hline & $u_{11}$ & $u_{28}$ & $u_{33}$ & $u_{12}$ & $u_{13}$ & $u_{23}$ \\
\hline & $144 \quad(3)$ & $165 \quad(3)$ & 0.0207 & .0006 & $0.0019(2)$ & 0.0012 \\
\hline & $4 \quad(5)$ & 0.0 & 8 & & -0 & 40 \\
\hline & $65 \quad(5)$ & 0.0 & $2 \quad(5$ & & -0 & 36 \\
\hline & & 0.0 & & & & \\
\hline & (12) & 0.0 & 10 & $3(1)$ & $9(9)$ & 16 \\
\hline & 0 & & & & $12(9)$ & 0 \\
\hline
\end{tabular}

In Tables 1, 2, and 3, numbers in parenthesis are standard deviations in units of the last digit.

Table 2. Interatomic distances in $\AA$, and angles in degrees in the $\mathrm{H}_{2} \mathrm{SeO}_{3}$ molecule.

Uncorrected distance

$$
\begin{aligned}
& \mathrm{Se}-\mathrm{O}(1) \\
& \mathrm{Se}-\mathrm{O}(2) \\
& \mathrm{Se}-\mathrm{O}(3) \\
& \mathrm{O}(2)-\mathrm{H}(1) \\
& \mathrm{O}(3)-\mathrm{H}(2) \\
& <\mathrm{O}(1)-\mathrm{Se}-\mathrm{O}(2) \\
& <\mathrm{O}(1)-\mathrm{Se}-\mathrm{O}(3) \\
& <\mathrm{O}(2)-\mathrm{Se}-\mathrm{O}(3) \\
& <\mathrm{Se}-\mathrm{O}(2)-\mathrm{H}(1) \\
& <\mathrm{Se}-\mathrm{O}(3)-\mathrm{H}(2)
\end{aligned}
$$

Distance corrected for riding motion, the light atom riding on the heavy atom

A list of observed and calculated structure factors is given in Table 4.

Fig. 1 is a projection of the structure along the $y$ direction. The drawing is done by the programme ORTEP. ${ }^{19}$ 
Table 3. Distances in $\AA$, and angles in degree in the hydrogen bonds. Different marking of the atoms indicates atoms from different molecules.

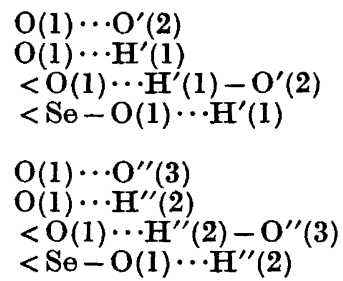

$\begin{array}{cc}2.667 & (2) \\ 1.749 & (3) \\ 153.36 & (27) \\ 130.56 & (13) \\ & \\ 2.621 & (2) \\ 1.621 & (3) \\ 170.56 & (27) \\ 121.64 & (13)\end{array}$

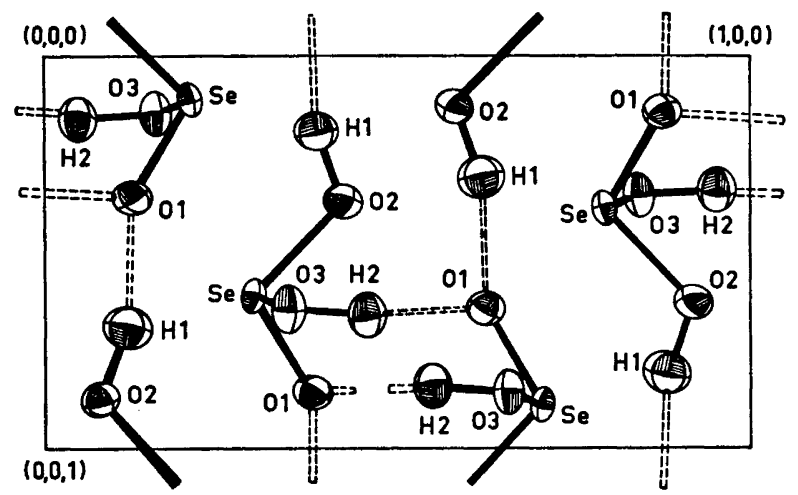

Fig. 1. Projection of the structure along the $y$ direction. Dotted lines between atoms, hydrogen bonding. Broken lines, bonding to atoms in neighbouring unit cells.

\section{DISCUSSION}

The arrangement of the selenite groups, $\mathrm{SeO}_{3}$, and the positions of the hydrogen bonds are as found by Wells and Bailey. However, the distances within the selenite group are somewhat different from those found in the previous determination. The most important new result is the location of the hydrogen atoms.

The $\mathrm{Se}-\mathrm{O}$ distances fall into two groups, a short $\mathrm{Se}-\mathrm{O}(1)$ distance (1.64 $\AA)$ for the oxide-oxygen, and two longer distances, $\mathrm{Se}-\mathrm{O}(2)(1.74 \AA)$ and $\mathrm{Se}-\mathrm{O}(3)(1.74 \AA)$, for the hydroxyl-oxygens, the hydrogen atoms being bonded to $\mathrm{O}(2)(0.99 \AA)$ and $\mathrm{O}(3)(1.01 \AA)$. The difference between the two $\mathrm{O}-\mathrm{H}$ distances is $0.023 \AA$ in the uncorrected case, and $0.019 \AA$ when the distances are corrected for "riding motion" (Busing and Levy ${ }^{20}$ ). The corresponding differences for the $\mathrm{Se}-\mathrm{O}(2)$ and the $\mathrm{Se}-\mathrm{O}(3)$ distances are $0.008 \AA$ and $0.004 \AA$. The standard deviation is $0.004 \AA$ for the difference between the two $\mathrm{O}-\mathrm{H}$ distances, and $0.003 \AA$ for the difference between the two $\mathrm{Se}-\mathrm{O}$ distances. The hypothesis that the two $\mathrm{O}-\mathrm{H}$ distances are identical, can easily be rejected on a $5 \%$ significance level, assuming Gaussian distribution for the difference. A corresponding comparison for the $\mathrm{Se}-\mathrm{O}$ distances leads to the same result for the uncorrected case, but not for the corrected distances.

Acta Chem. Scand. 25 (1971) No. 4 
Table 4. Observed and calculated structure amplitudes included in the refinements.

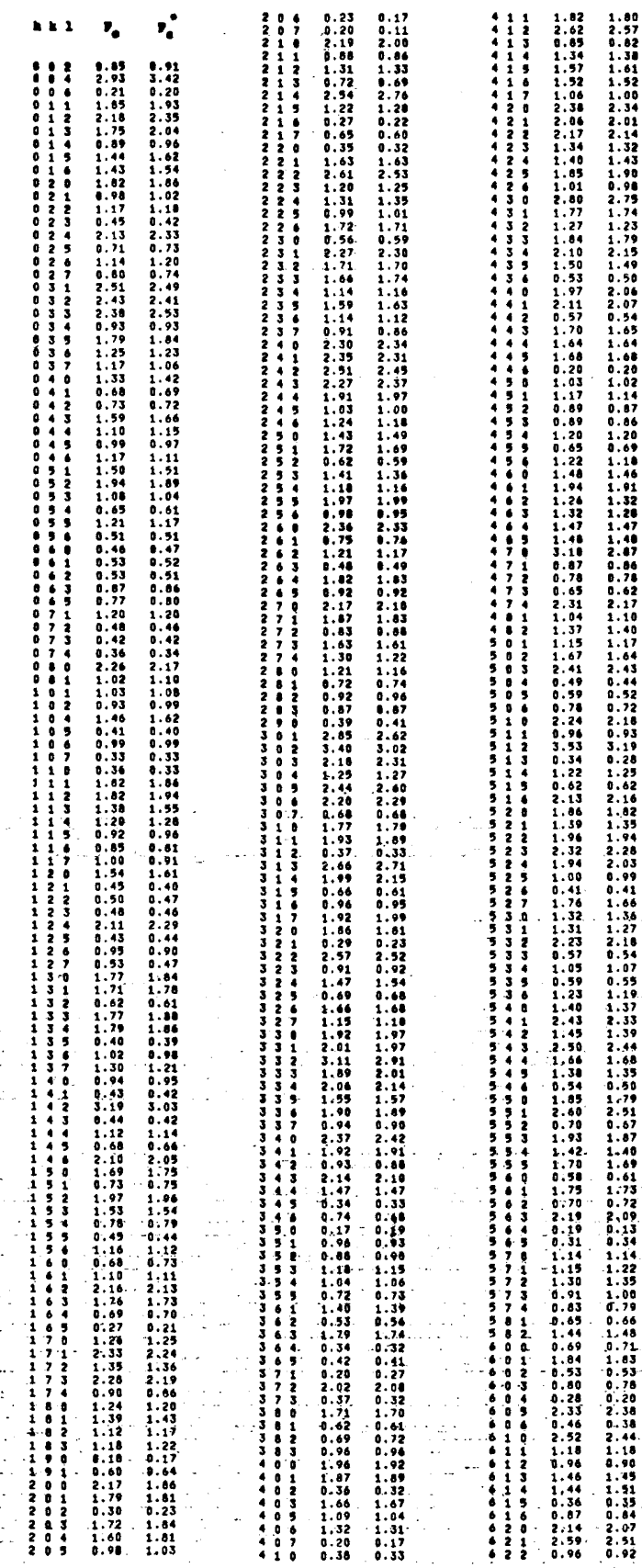

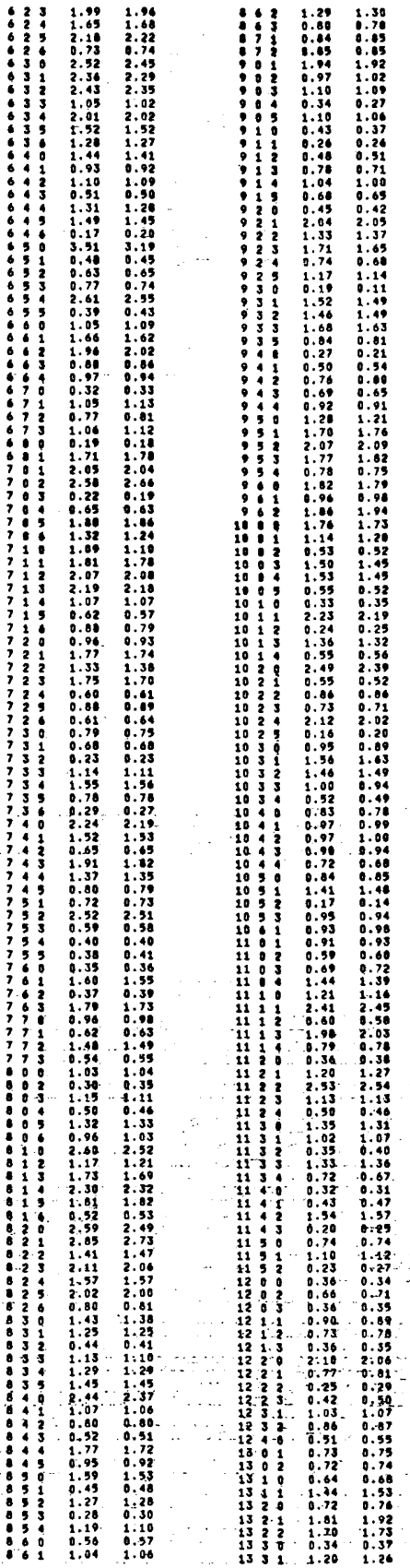

Acta. Chem. Scand. 25 (1971) No. 4 
We must therefore conclude that the two $\mathrm{O}-\mathrm{H}$ distances are significantly different, whereas this is not the case for the two $\mathrm{Se}-\mathrm{O}$ distances.

The angles $<\mathrm{O}(1)-\mathrm{Se}-\mathrm{O}(2)$ and $<\mathrm{O}(1)-\mathrm{Se}-\mathrm{O}(3)$ differ by $8.1^{\circ}$, so in the crystalline state the $\mathrm{SeO}_{3}$ part of the molecule does not have the $C_{3}$ symmetry, which would be assumed for the "free state" of the molecule.

The coordination of the selenium atom is distorted octahedral (the three corners of the octahedron being the oxygen atoms in the molecule), when oxygen atoms near lines defined by $O(1)-S e, O(2)-S e$, and $O(3)-S e$ are taken into consideration. These Se-O distances are 2.957 (2) $\AA, 3.240$ (2) $\AA$, and 3.079 (2) $\AA$, respectively.

Each $\mathrm{SeO}_{3}$ group is held in position by four hydrogen bonds.

The oxide-oxygen, $\mathrm{O}(1)$, is the end point of two hydrogen bonds, the other end points of the two bonds being $O(2)$ and $O(3)$ in two different molecules.

If this $\mathrm{H}(1) \cdots \mathrm{O}(1) \cdots \mathrm{H}(2)$ configuration, which constitutes the hydrogen bonding system is thought of as a molecular entity $(\mathrm{HOH})$ with $\mathrm{O}-\mathrm{H}$ bond lengths 1.749 and $1.621 \AA$, and bond angle $94.1^{\circ}$, the bonding in the structure can be described generally in the following way: the negative end of the $\mathrm{HOH}$ entity (i.e. the oxygen atom) is attached to the positive end of the $\mathrm{SeO}_{2}$ configuration (i.e. the selenium atom), whereas the positive parts of $\mathrm{HOH}$ (i.e. the two hydrogen atoms) are attached to the negative parts in two $\mathrm{SeO}_{2}$ groups (i.e. the two oxygen atoms).

The hydrogen bonded framework is a two-dimensional double layer parallel to the (100) plane, with one hydrogen atom $(\mathrm{H}(1))$ involved in bonding in the $z$ direction, and the other hydrogen atom $(\mathrm{H}(2))$ involved in both bonding in the $y$ direction and in interlinking of the two layers (Fig. 1).

The length of the two hydrogen bonds differ little. The longest bond $\mathrm{O}(1) \cdots \mathrm{O}(2)$ is "bent" $20^{\circ}$ more than the shortest bond $\mathrm{O}(1) \cdots \mathrm{O}(3)$, and correspondingly the $\mathrm{O}(2)-\mathrm{H}(1)$ distance is shorter than the $\mathrm{O}(3)-\mathrm{H}(2)$ distance. The shortest distance between two hydrogen atoms is $2.469 \AA$.

Infrared and Raman studies on the $\mathrm{H}_{2} \mathrm{O}-\mathrm{SeO}_{2}$ system (i.e. $\mathrm{H}_{2} \mathrm{SeO}_{3}$ ) have been reported in several articles (Detoni and Hadzi, ${ }^{21}$ Falk and Giguere, ${ }^{22}$ Simon and Paetzold ${ }^{23}$ ). For a hydrogen bond with a length of $2.64 \AA$, the expected $\mathrm{O}-\mathrm{H}$ stretching frequency would be about $2650 \mathrm{~cm}^{-1}$ (Nakamoto et $\left.a .^{24}\right)$.

Two $\mathrm{OH}$ stretching frequencies are observed at $2300 \mathrm{~cm}^{-1}$ and $2900 \mathrm{~cm}^{-1}$, respectively. There are two independent hydrogen bonds, but their differences in bonding angle etc. are probably not large enough to explain the size of the splitting of the stretching frequency.

Simon and Paetzold suggest that the splitting results from tunneling of the proton between two minima of potential energy in the hydrogen bond. However, a difference map, calculated at the end of the refinement, gives no indication of the presence of protons outside the positions already determined. Blinc et al..$^{25}$ place the potential function in the hydrogen bond of selenious acid in the group of proton potential curves which are "practically equal to (asymmetric) single minimum ones". Giguere and Falk explain the splitting as resulting from a "strong coupling of the two $\mathrm{OH}$ groups through the heavy selenium atom". Consideration of the structure, as determined here, indicate 
that coupling of the two $\mathrm{O}-\mathrm{H}$ groups through the $\mathrm{O}(1)$ atom, which is bonded to both hydrogen atoms, is also possible. This coupling seems to be as good an explanation for the splitting as the coupling through the selenium atom.

Acknowledgements. We wish to thank Professor S. E. Rasmussen for helpful assistance and discussion throughout the investigation. Statens Almindelige Videnskabsfond, and the Danish Atomic Energy Commission are thanked for the financial assistance towards the purchase and maintenance of the Hilger-Ferranti neutron diffractometer.

\section{REFERENCES}

1. Wells, A. F. and Bailey, M. J. Chem. Soc. 19491282.

2. Hansen, F., Hazell, R. G. and Rasmussen, S. E. Acta Chem. Scand. 23 (1969) 2561.

3. Schousboe-Jensen, V. H. F. Algol program $S_{1}$. Printed in this laboratory.

4. Arndt, U. W. and Willis, B. T. M. Single Crystal Diffractometry, The University Press, Cambridge 1966.

5. Lehmann, M. S. and Larsen, F. K. Algol program DRAM. Printed in this laboratory.

6. Wells, M. J. Acta Cryst. 13 (1960) 722.

7. Bhuiya, A. K. and Stanley, E. Acta Cryst. 16 (1963) 881.

8. Danielsen, J. Algol program $D 445$. Printed in this laboratory.

9. Busing, W. R., Martin, K. O. and Levy, H. A. ORFLS IUCC World List No. 363.

10. Stewart, J. M. $X$-ray 63, University of Maryland.

11. Hazell, R. G. Algol program G403. Printed in this laboratory.

12. Zachariasen, W. H. Acta Cryst. 16 (1963) 1138.

13. Larson, A. C. Acta Cryst. 23 (1967) 664.

14. The Neutron Diffraction Commission. Acta Cryst. A 25 (1969) 391.

15. Brown, G. M. and Chidambaram, R. Acta Cryst. B 25 (1969) 676.

16. Columinas, C. Phys. Rev. 153 (1967) 558.

17. Andresen, A. F. Acta Chem. Scand. 22 (1968) 827.

18. Fuess, H. and Will, G. Z. anorg. allgem. Chem. 358 (1968) 125.

19. Johnson, C. K. ORTEP, ORNL-3794. Oak Ridge National Laboratory, Oak Ridge, Tennessee.

20. Busing, W. R. and Levy, H. A. Acta Cryst. 17 (1964) 142.

21. Detoni, S. and Hadzi, D. J. Chim. Phys. 53 (1956) 760.

22. Falk, M. and Giguere, P. A. Can. J. Chem. 36 (1958) 1680.

23. Simon, A. and Paotzold, R. Z. anorg. allgem. Chem. 301 (1959) 246.

24. Nakamoto, K., Margoshes, M. and Rundle, R. E. J. Am. Chem. Soc. 77 (1955) 6480.

25. Blinc, R., Hadzi, D. and Novak, A. Z. Elektrochem. 64 (1960) 567.

Received September 3, 1970. 